\section{$\underset{\substack{\text { hommes } \\ \text { \& migrations }}}{ }$}

\section{Hommes \& migrations}

Revue française de référence sur les dynamiques

migratoires

$1306 \mid 2014$

Ecriture et migration

\title{
Faire de sa vie une fiction
}

Des migrations en écriture avec des enfants d'école élémentaire

\section{Bruno Hubert}

\section{(2) OpenEdition}

1 Journals

\section{Édition électronique}

URL : http://journals.openedition.org/hommesmigrations/2792

DOI : 10.4000/hommesmigrations.2792

ISSN : 2262-3353

Éditeur

Musée national de l'histoire de l'immigration

\section{Édition imprimée}

Date de publication : 1 avril 2014

Pagination : 23-30

ISBN : 978-2-919040-27-8

ISSN : 1142-852X

\section{Référence électronique}

Bruno Hubert, «Faire de sa vie une fiction », Hommes \& migrations [En ligne], 1306 | 2014, mis en ligne le 01 avril 2017, consulté le 19 avril 2019. URL : http://journals.openedition.org/

hommesmigrations/2792; DOI : 10.4000/hommesmigrations.2792 


\title{
FAIRE DE SA VIE UNE FICTION \\ DES MIGRATIONS EN ÉCRITURE AVEC DES ENFANTS D'ÉCOLE ÉLÉMENTAIRE
}

par BRUNO HUBERT, docteur en sciences de l'éducation, université de Nantes, formateur à l'École supérieure du professorat et de l'éducation Espe, chercheur associé au Centre de recherche en éducation (Cren).

\author{
Le récit d'une histoire personnelle et d'un parcours migratoire \\ n'est pas chose simple. Dans une classe de CM2 composée \\ de 23 élèves, dont la moitié d'origine étrangère, les mises \\ en situation proposées aux enfants leur permettent d'exprimer \\ leur singularité. En se racontant à l'école, en évoquant leur \\ histoire de vie par le détour de la fiction, les enfants peuvent \\ à la fois se l'approprier et la mettre à distance. \\ L'écriture offre ainsi un moyen de se situer et de poursuivre \\ une migration par l'imagination.
}

Écrire pour mettre en mouvement, pour faire bouger son regard sur les événements du passé ; cette association de l'écriture et du déplacement se retrouve dans toutes mes recherches antérieures, qu'il s'agisse pour les enfants d'explorer leurs propres cahiers d'école afin de prendre conscience des dynamiques de leur parcours ${ }^{1}$ ou, par le biais de l'écriture, de transformer leur vécu professionnel

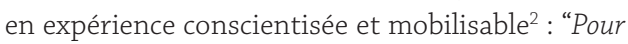
qu'un sujet s'affirme comme tel, il faut qu'il y ait une situation dans laquelle il puisse exister sous la forme d'une prise de parole." Dans la recherche dont il est question ici, l'objectif était d'abord de poursuivre le développement d'un modèle d'investigation dans son rapport au savoir visant, par l'écriture, la réinscription d'une histoire scolaire dans une histoire de vie, dans un mouvement d'autoconnaissance et dans une perspective à long terme conciliant école et vie, apprentissage et personne, société et intimité du sujet. Mais de par la population concernée : une classe de cours moyen deuxième année composée de 23 enfants, dont la moitié d'origine étrangère (dix pays différents représentés), les récits en lien avec la migration vont occuper une place très importante, si bien que nous nous sommes intéressés aux liens qui se tissent entre le travail de l'histoire de vie en contexte scolaire et l'écriture de la migration. 


\section{La possibilité de se dire à l'école}

Cette recherche trouve sa justification dans le constat d'impuissance formulé par l'enseignante d'un CM2 de Coulaines - ville qui jouxte Le Mans -, face aux difficultés éprouvées par ses élèves, notamment ceux d'origine étrangère, pour faire une place aux apprentissages scolaires dans leur vie personnelle. Beaucoup de ces enfants rencontrent, par ailleurs, de réels obstacles quand il leur faut manier la langue française à l'écrit. Pour eux, l'école reste un monde à part, comme ne faisant pas partie de la vraie vie: "Celle où l'on apprend le monde, les autres et soi, se déroule à l'extérieur de ses murs"."

Partant de l'idée que l'insertion scolaire passe par l'instauration d'un rapport au langage, il s'agit d'abord d'explorer l'utilisation de l'écriture comme une forme d'action, de dialogue avec soi et avec l'autre, comme instrument pour penser ${ }^{5}$. La lecture et l'écriture ne peuvent pas être définies simplement comme des compétences-outils détachées de soi : elles sont le sujet. L'imbrication des niveaux cognitif, psycho-socio-affectif et langagier nous incite à impliquer la personne-élève dans les situations d'écriture pour qu'elle puisse donner forme à ses migrations identitaires. Différentes situations d'écriture ont été proposées aux enfants, par petits groupes de six, à raison d'une fois par semaine, dans des séances d'une durée n'excédant pas trente minutes, pour déclencher l'expression individuelle des parcours singuliers ${ }^{6}$, notamment à partir de traces du passé : photographies, objets personnels, écrits scolaires antérieurs conservés par les sujets? Les phases orales enregistrées avec l'accord des enfants et les écrits produits ont été ensuite analysés avec une démarche qualitative et impliquée, cette recherche se situant dans le champ phénoménologique et herméneutique ${ }^{8}$ et, plus précisé- ment, selon l'approche biographique-narrative et les méthodologies qui lui correspondent.

Pour la première séance, il avait été demandé aux enfants d'apporter une photographie personnelle à laquelle ils tenaient. Ce qui a d'abord frappé l'enseignante, c'est l'enthousiasme avec lequel les enfants ont majoritairement répondu à la consigne et le caractère très intime de ce qu'ils ont ramené ; certaines familles avaient même confié leur album pour que leur enfant puisse parler d'une photo qui y était rangée. Pour ne pas d'emblée placer les enfants en position difficile par rapport à l'écrit, chacun devait d'abord présenter sa photo oralement aux cinq autres membres du groupe et à l'animateur, puis expliquer pourquoi il l'avait choisie. Afin d'illustrer ce qui s'est joué dans ce premier moment, nous prendrons l'exemple de l'entretien réalisé avec Jérémy, qui avait apporté une photographie ressemblant à une photo d'identité à propos de laquelle il lui paraissait difficile de s'exprimer.

Animateur: Qu'est-ce que c'est cette photo?

Jérémy : C'était la photo la première fois...

(incompréhensible)

A. : Je n'ai rien compris avec ton chewing-gum...

J. : C'était la photo de mon école.

A. : C'était la photo de ton école?

J. : Pas ici.

A. : T'étais où?

J. : J'aime pas trop en parler... mon pays...

A. : T'étais dans ton pays?

J. : En Afrique.

A. : C'est pas un pays l'Afrique?

J. : Au Congo.

A. : C'était ta photo d'école au Congo?

Jérémy: Ouais... C'était en CE1.

A. : T'es arrivé en France pour le CE2 ?

Madina : Moi aussi.

A. : Quand tu es arrivé en France, tu ne savais pas du tout le français?

J. : Rien.

3. Michel Agier, "Le sujet dans la cité, un espace de controverse”, in Le Sujet dans la cité, n 1, 2010. 4. Jean-Michel Baudouin, Laurence Türkal, "Formations au singulier", in Éducation permanente, n¹42, 2001, p. 54. 5. Élisabeth Bautier, Dominique Bucheton, "Interactions : co-construction du sujet et des savoirs", in Le Français aujourd'hui, n 113, 1996, pp. 24-32. 6. Christine Delory-Momberger, La Condition biographique. Essai sur le récit de soi dans la modernité avancée, Paris, Téraèdre, 2009. 7. Bruno Hubert, Faire parler ses cahiers d'écolier, op. cit. 8. Paul Ricœur, Du texte à l'action. Essais d'herméneutique, t. II, Paris, Seuil, 1986. 

A. : Tu as déjà appris beaucoup de choses.
J. : Pas trop.

A. : Tu trouves que tu n'as pas appris assez de choses. Tu as l'air de te débrouiller quand même...

Qu'est-ce que tu peux nous dire sur cette photo?

J. : C'était pas une école normale comme les autres écoles.

A. : C'était pas une école normale? (...)

J. : On se battait... plus qu'ici en France...

A. : Pourquoi?

J. : On est trop...

A. : Vous étiez beaucoup à l'école?

J. : On est vers cent... On était trop...

A. : Pourquoi es-tu venu en France?

J. : Pour faire mes études... (silence) non pas pour faire mes études... mais pour voir mon père qui habite ici...

A. : C'est quoi la langue au Congo?

$\mathrm{J}$ : : Ah, je me rappelle plus.

A. : C'est incroyable cela... Tu sais toujours la parler?

J. : Oh là là, c'est dur ! ... lingala voilà...

A. : Est-ce que tu peux nous dire une phrase?

J. : ... J'ai la honte.

A. : Pourquoi tu as la honte? C'est bien de parler deux langues...

$\mathrm{J}$. : Aux grandes vacances, je vais y aller...

Peut-être je vais rester là-bas, on sait jamais.

A. : Tu aimerais bien rester là-bas?

J. : Ouais. C'est mon pays...

C'est là-bas que je suis né, je suis habitué...

A. : Tu te sens pas chez toi ici?

J. : Y a pas assez de sable...

Madina : Je peux le refaire moi?

\section{S'autoriser à l'expression}

Cet entretien constitue une très bonne illustration de ce qui va s'enclencher. D'abord le désir de parler des origines : tous les enfants concernés par la migration apporteront une photographie ayant un rapport avec leurs origines et avec la sphère fami- liale. J'ai déjà constaté dans mes travaux précédents ${ }^{9}$ que même à l'intérieur de l'école, la famille semble jouer un rôle essentiel dans l'acquisition des savoirs. Pour Vincent de Gaulejac, "la famille est le lieu privilégié de la transmission"10". Mais ils montreront une véritable envie de s'exprimer sur leur histoire personnelle, auront toujours peine à croire que c'est bien dans le cadre de l'école que cette possibilité leur est offerte.

Jérémy commencera par marmonner et prononcer une phrase incompréhensible, puis il dira: "J'aime pas trop en parler... mon pays...", alors que personne ne l'a obligé à choisir cette photographie. D'autres demanderont au début de leur présentation s'ils ont bien le droit de parler de "ça", comme si la famille, les racines étrangères et l'école ne pouvaient pas se rencontrer. L'intervention de Madina, qui clôt l'entretien de Jérémy, est à cet égard Pour ne pas d'emblée placer les enfants en position difficile par rapport à l'écrit, chacun devait d'abord présenter sa photo oralement aux cinq autres membres du groupe et à l'animateur, puis expliquer pourquoi il l'avait choisie.

juste avant et a décrit une photographie de la fête de l'Aid lorsqu'elle habitait Djibouti. Manifestement, Madina n'a pas tout dit et, lorsqu'elle entend Jérémy, elle s'immisce une première fois pour signaler qu'elle aussi est arrivée en CE2 et quand Jérémy a terminé, elle émet le désir de s'exprimer à nouveau, d'ajouter quelque chose, ce qu'elle fera d'ailleurs dans l'écrit qui suivra : "Il y a beaucoup de ma famille à Djibouti mais ils ne veulent pas venir en France. J'ai même un deuxième nom : 'Asline'. Je peux apprendre par ma mère et mon père la langue arabe et djiboutienne." Son écrit s'achève ainsi, par des éléments non présents à l'oral, que le témoignage de Jérémy a en quelque sorte autorisés.

De même, la question des langues apparaît très sensible, elle sera évoquée dans de nombreux écrits, par exemple par Ana: "J'ai pris cette photo parce que c'est la première nièce que je l'ai eue. Je la vois pas tout le temps parce que ma belle-sour, elle sait pas parler français. Lorsqu'elle parle portugais comme 
moi, bah des fois je lui apprends à parler français. J'aide aussi maman." De la petite nièce, on passe très rapidement à cette problématique des langues, sur laquelle Ana semble avoir très envie de communiquer. En ce qui concerne Jérémy, celui-ci vit mal sa non-maîtrise du français à l'écrit, à tel point qu'il a l'impression de ne pas avoir Le fait que la parole appris grand-chose depuis puisse revenir sur ce qui son arrivée : "pas trop".

constitue pour l'enfant Le fait de parler une autre un bouleversement ne rend pas ces mutations plus faciles, mais cela permet de leur donner corps, de ne pas les nier, de commencer à accepter leur réalité. dur" pour lui de prononcer son nom et quelques mots, comme s'il fallait cacher cette partie honteuse de lui. D'ailleurs, le terme de "normal" est attaché à lécole française : "C'était pas une école normale comme les autres écoles", l'école du Congo étant associée à la violence : "On se battait... plus qu'ici en France..." et au nombre : "On est trop." Remarquons que Jérémy passe pourtant de l'imparfait au présent, ce qui donne l'impression qu'il y est encore. Il envisage un retour possible : “C'est mon pays... C'est là-bas que je suis né, je suis habitué..."

\section{Donner une forme écrite au mal-être}

L'écrit qui suivra sera une épreuve pour Jérémie, mais il parviendra au prix de dix minutes d'efforts à produire le texte suivant, qui fait écho à l'échange qui a précédé : "Dans mon péi, dans mon école, cent enfants en bagarre. Il y a plein de sable. Je vais retourner dans mon péi, il sappelle Congo." Peu importe que ce propos soit fondé, car le "on ne sait jamais" dont il est assorti à l'oral permet d'avoir quelques doutes sur l'éventualité d'un retour, mais la mise à l'écrit de cette aspiration transcrit la difficulté qu'éprouve Jérémy à évoluer d'un monde à l'autre. Où et quand dire ce malaise alors que, sous couvert d'intégration, chacun va justement s'appliquer à ne pas faire référence à ce vécu antérieur, à ne pas mentionner, et notamment dans le cadre de l'école, les embûches et les mises en danger liées à la migration ? Où et quand dépasser les discours convenus: "Je suis venu en France pour faire mes études..."?

Le fait que la parole puisse revenir sur ce qui constitue pour l'enfant un bouleversement ne rend pas ces mutations plus faciles, mais cela permet de leur donner corps, de ne pas les nier, de commencer à accepter leur réalité. Jérémy comme Madina auront d'ailleurs besoin de revivre plusieurs fois en quelque sorte cette inscription dans l'écriture comme pour mieux réaliser eux-mêmes le processus en cours. Quand il s'agira d'apporter un objet auquel on tient, ce que José González Monteagudo pratique en formation à l'université de Séville ${ }^{11}$, Madina choisira un cadre avec une photographie de son grand-père, mais ce cadre ne sera pas matériellement présent car il est resté avec certains membres de la famille dans la maison de Djibouti. Quant à Jérémy, il optera pour un cadre qui trône dans l'appartement où il habite en France, mais la photographie embrasse toute sa famille du Congo devant la maison de là-bas : "J'ai une photo de ma famille qui vien de mon pay le Congo. Ses une photo de moi et mon pere et maman et ma tata et tonton et mon cousin et neveu. C'est de vant la maison."

Lesproblèmes desegmentation que Jérémy connaît dans son écriture du français ne sont-ils pas liés à cette coupure qui s'est opérée dans sa vie ? Ainsi sort-elle du silence pour exister dans le langage et, qui plus est, dans la langue du pays où il faut maintenant se projeter. Progressivement s'installe chez l'enfant l'intuition qu'il a quelque chose à dire et qu'il peut engager la quête d'une position subjective $^{12}$. 


\section{Réécrire son histoire}

Inscrire son histoire dans les courbes de l'écriture pour vivre intensément les virages de sa vie, c'est ce qui constitue l'objectif de la présente recherche. Cela me rappelle un roman de l'écrivain italien Alessandro Baricco, dont le titre français est précisément Cette histoire-là. Dans ce roman, Elizaveta transforme sa vie en aventures rocambolesques et laisse traîner son journal pour que son collègue de travail avec qui elle trace la route le lise: "C'était pour Ultimo que jécrivais tout cela, je le sais bien. J'oubliais mon journal partout, tous les jours il le lisait et le remettait en place. Il ne m'en a jamais rien dit. Mais je savais qu'il le lisait. Nous avions ces deux jeunesses recluses, cette sorte d'exil insensé, et tout ce que nous pouvions faire, cétait imaginer ce que nous n'avions pas. Des histoires. Lui, il avait sa piste, au milieu de nulle part, avec toutes les courbes qu'il avait dérobées au monde. Moi, j'écrivais pour lui. Pour moi. Qui sait ${ }^{13}$ ?"

Elizaveta ira jusqu'à raconter dans son journal qu'Ultimo est parti et il partira un jour sans rien dire, donnant ainsi réalité à la fiction: "Je sais maintenant que c'est une des plus belles choses que j’ai faites. Ces mois avec Ultimo à trimbaler des pianos. Et le soir à écrire pour lui. Quelquefois je réécrivais les histoires qu'il m'avait racontées. J'aimais le transformer en personnage de roman, en invention. Je voulais qu'il sache qu'il était quelqu'un de spécial, comme on en lit dans les livres, comme il en lisait, lui, dans ses bandes dessinées. Un héros. Voilà, je voulais peut-être qu'il sache qu'il était un héros ${ }^{14}$." Alex Lainé a exploré les liens de parenté entre littérature et histoire de vie, l'entreprise romanesque étant toujours de créer la vie, d'inventer des personnages et de les doter d'une histoire : "Il n'est pas impossible que l'écriture romanesque et la production de son histoire de vie par un sujet procèdent de la même source, du même désir originaire : celui, sinon de créer ou de "produire sa vie", du moins d'avoir prise sur elle $e^{15}$."

\section{Faire de l'autre un personnage de roman}

À l'image d'Elizaveta et d'Ultimo, des situations ont été proposées aux enfants pour qu'ils écrivent à partir de ce que l'autre avait raconté de sa vie. Par exemple, quand on leur a demandé d'apporter un objet important, Quentin est venu avec trois singes qui s'empilaient les uns sur les autres, il a expliqué à l'oral pourquoi il avait fait ce choix ; ensuite, c'est Roman qui a écrit un petit texte sur ce qu'avait dit

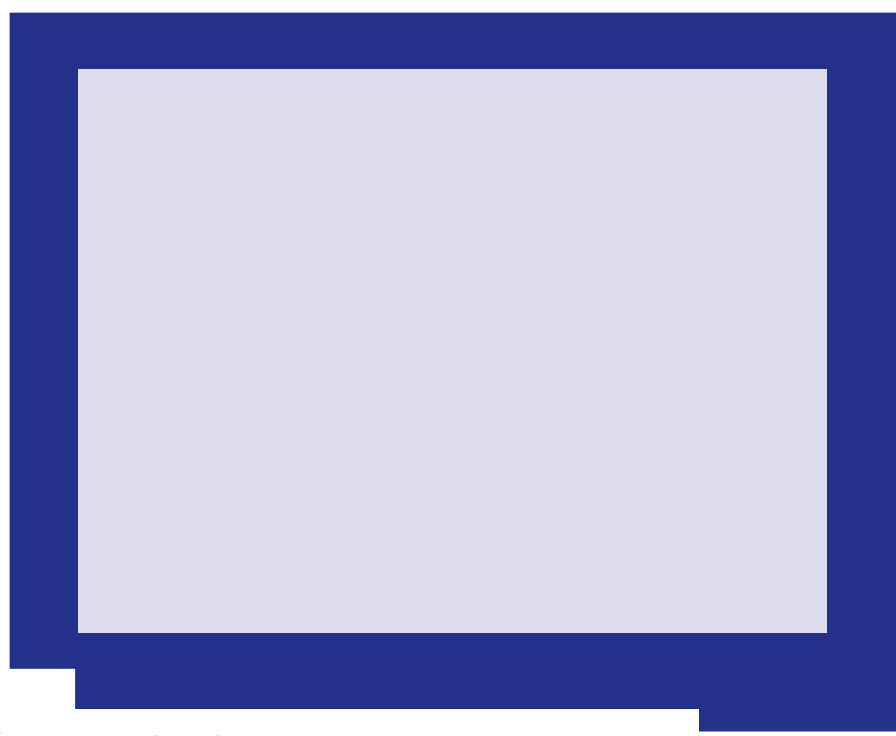

(c) Henri Bokilo-Boursier, 2014

son camarade et inversement: "Quentin a emmené des singes : un qui est sourd, un qui ne voit pas et un qui n'a pas la parole, c'est son grand-père qui les a fabriqués en bois. Il les a vus depuis qu'il est né, il les met devant sa télévision. Il a quelquefois l'impression de ressembler à celui qui ne parle pas, car s'il s'exprime 
à l'oral, il a beaucoup de mal à écrire." En quelque sorte, par le biais des singes, Quentin a pu dire combien lui pesait le passage à l'écrit. En transformant la parole en un petit récit où Quentin est peu à peu repris par le pronom personnel "il”, Ronan donne une certaine dimension à ce personnage qui trouve une façon imagée, presque métaphorique, de pointer ses difficultés d'écriture que bon nombre d'entre eux au sein du groupe partagent: "Lêtre qui est au centre d'une histoire de vie, celui qui est, d'une certaine manière, le héros, ne doit être tenu ni pour un objet absolu, ni pour un sujet absolu, mais comme une sorte d'intermédiaire entre les deux ${ }^{16}$." Quand Coralie écrira ce que Madina a raconté à propos du cadre qui met en valeur son grand-père, elle choisira une narration à la première personne, ce qui peut paraître étonnant: "J'ai une photo de mon grand-père. Mais elle est dans mon pays qui est Djibouti. Mon grand-père est décédé quand j'avais quatre ans le jour de l'Aid.

"Lêtre qui est au centre d'une histoire de vie, celui qui est, d'une certaine manière, le héros, ne doit être tenu ni pour un objet absolu, ni pour un sujet absolu, mais comme une sorte d'intermédiaire entre les deux." Etc'est la seule photo que j'ai de lui grand." Par le truchement de l'écriture, Coralie se met d'autant plus facilement en empathie avec Madina que pour la séance à partir d'une photographie personnelle, elle avait apporté une photographie prise à l'occasion du mariage d'une tante et d'un oncle en Algérie où, âgée de 2 ans, elle porte un costume rouge à paillettes. De son côté, Madina rédigera facilement un petit texte à partir de l'objet sélectionné par Coralie, elle restera d'ailleurs dans l'ambiguité d'une troisième personne non élucidée: "C'est un genre de tasse où il y a un chat dedans. Elle l'a eue le jour de ses 10 ans. Sa tata lui a offerte quand elle est venue en France, elle l'a achetée en Algérie et pour elle ça compte beaucoup à ses yeux." Comme dans un roman, chacun retrouve des portions de son histoire dans celle du pair et le "ça compte beaucoup à ses yeux" n'a jamais été formulé comme tel par Coralie. C'est donc à la fois l'histoire de Coralie que développe Madina, mais aussi la sienne, et, en même temps, ni totalement celle de Coralie ni totalement la sienne, mais une fiction qui puise dans leurs vécus respectifs.

\section{Concilier les multiples temporalités}

Un double mouvement d'actualisation/distanciation s'opère chez les enfants vis-à-vis de l'imaginaire des origines étrangères. D'une part, l'écriture rend présents, visibles, les fragments du passé que le sujet s'approprie et recompose par le travail d'histoire de $v \mathrm{e}^{17}$, d'autre part, cette mise en mots, parce qu'elle ouvre la voie à l'invention, accompagne le déplacement de la personne dans un mouvement de conciliation des multiples temporalités ${ }^{18}$. Dans le texte produit par Coralie, le présent s'applique aussi bien à la photographie du grand-père qu'à Djibouti, alors que celui-ci est décédé il y a longtemps déjà : “J'avais 4 ans". Cette interdépendance des différentes strates temporelles légitime de rapprocher en formation, ainsi que le suggérait Gaston Pineau au travers de son concept de "chronoformation" ${ }^{19 "}$, les temps disparates, des temps qui fonctionnent souvent par couples opposés : temps familial/temps de l'école, temps de l'enfant/temps de l'élève, temps du souvenir/temps du présent, temps de l'étranger/ temps de la France, temps des adultes/temps des enfants... Ce travail de mise en résonance des temporalités qui, comme "le temps imaginaire échappe à la contingence chronologique ${ }^{20 "}$, ouvre les mondes internes à d'autres significations qui permettent au sujet non pas de changer le passé, mais d'en reconstruire le sens. Coralie n'est pas, elle, d'origine étrangère, elle a juste de la famille en Algérie, mais l'écriture lui offre la possibilité de se recon-

16. Ibid., p. 77. 17. Jean-Louis Le Grand, “Définir les histoires de vie. Sus et insus définitionnels”, in Revue internationale de psychosociologie, n 14 2000. 18. Bruno Hubert, Fred Poché, “La formation face aux défis de la responsabilité”, in Éducation permanente, $n^{\circ} 187,2011$. 
naître dans les sentiments d'appartenance familiale développés par Madina ; à l'inverse, Madina voit ce qu'elle a dit de sa propre histoire fixé par une autre dans l'écriture.

Abdelkarim, lui, a choisi comme objet important un mini-Coran ; il était à la fois très fier de le montrer et un peu inquiet de la réaction quallaient avoir l'animateur et ses camarades. Il a ouvert le livre et a expliqué que dans ce Coran pour enfants les phrases en arabe étaient traduites en français et aussi en phonétique. Abdelkarim se sert de la phonétique pour lire l'arabe. Les autres lui ont demandé de lire quelques passages en arabe. Puis c'est Lucas, non musulman, qui a écrit à partir de ce qu'avait dit Abdelkarim : "Abdel a ramené le livre du Coran. C'est très instruisant. Il y a trois langues différentes : en arabe, français, phonétique. Il lit en rouge et sait lire en phonétique." Ce qui est à noter, c'est que les pairs ont d'abord écouté Abdelkarim avec attention; à aucun moment il n'y a eu de remarques négatives vis-à-vis de la religion, alors que le groupe était très mélangé sur ce plan. Ensuite, c'est surtout sur l'accession à la langue arabe que se sont focalisées les questions. D'ailleurs, ce que retient Lucas, ce n'est pas qu'Abdelkarim ne sache pas lire l'arabe mais qu'il se débrouille avec la phonétique. D'une certaine manière, lui aussi devient "une sorte de héros" aux yeux de son camarade. Cela peut contribuer à aider Abdelkarim à assumer sa migration, lui dont la grand-mère habite à Oran mais qui a besoin du français pour accéder au Coran et qui a grandi en France. La photographie qu'il avait apportée précédemment le représentait au pied d'une statue de lion dans le centreville d'Oran ; pour Abdelkarim, ce passé algérien tient plus du mythe fondateur, des racines ancestrales, de la tradition familiale, que du quotidien, mais il importe pour lui d'inscrire sa vie actuelle et future dans le prolongement de ces histoires héritées et de ces histoires racontées sur lui-même. Le passage par lécriture, en prenant en compte la réalité du vécu, participe à son intégration tout en instaurant une distance.

\section{S'inventer par l'écriture}

Pour favoriser le métissage, les situations d'écriture proposées joueront de la mise en fiction ${ }^{21}$, en favorisant la réécriture, les réarrangements, qui aident à donner à voir, à entendre, à sentir, et qui peu à peu confèrent à l'écrivant un pouvoir sur ses histoires. Nous nous sommes inspirés des œuvres de l'artiste française Sophie Calle afin d'amener les enfants à entremêler leurs propres traces : photos, dessins, journaux, lettres... et celles des autres, dans le but de travailler sur leur vie.

Dans le livre Les Panoplies présent dans le coffret Doubles-jeux ${ }^{22}$, Sophie Calle a engagé un détective privé afin qu'il la suive ; elle utilise des morceaux réels Le passage parl'écriture, de sa vie en menant où elle en prenant en compte le souhaite le détective, elle la réalité du vécu, prend tout ce qui lui arrive participe à son intégration comme matériau d'écriture tout en instaurant et transforme son histoire une distance. en matière à raconter, en fiction potentielle. Pour lancer l'écriture, l'animateur avait produit un petit texte intitulé "Rapport de monsieur Kivoitout, détective privé", qui racontait une journée type de l'animateur, comme s'il avait été suivi par un détective. Ce texte a été lu dans chaque groupe avec les élèves et la situation de filature a été explicitée ; ensuite, chacun a dû imaginer que quelqu'un avait été payé pour le suivre et que celui-ci remettait un rapport du même type. Certains ont eu beaucoup de difficultés à abandonner le "je" et à parler d'eux à la troisième personne, ce qui demande un effort intellectuel de décentration, de prise de recul vis-à-vis de soi-même, mais c'est précisément ce déplacement qui s'avère intéressant: "Rapport de monsieur Boudin, détective privé, sur mademoiselle Joyce. Joyce habite dans un appartement à Coulaines rue de M. avec sa mère et son petit frère. Tous les matins, la petite famille se réveille à 7 heures. La nuit, la mère 
se lève pour faire des prières musulmanes. Joyce part à l'école vers 8 h 20. Elle sort de l'école à 16 h 30. Elle part chez sa grand-mère à côté de son école, il y a beaucoup de monde là-bas. Son père vient la chercher pour aller à Paris un week-end sur deux. Son grand-père a été hospitalisé et a été mis en maison de repos."

Ce qui frappe dans ce récit, c'est l'omniprésence de la famille : "sa mère", "son petit frère", "sa grandmère", "son grand-père", "son père", ce qu'on retrouvera dans beaucoup d'écrits. Pour construire leur identité comme leur rapport au savoir, les enfants ont besoin de cette reliance qu'installent les mots entre les différentes parties d'eux-mêmes. Sans ces liens, l'impression de perte de connaissance n'autorise pas à imaginer sa vie parmi les autres et à l'inscrire dans une temporalité. En quelques lignes, Joyce parvient à marquer sa filiation dans une culture musulmane, son inquiétude vis-à-vis de la santé de son grand-père et son quotidien d'enfant ayant des parents séparés et ne vivant pas dans la même ville. La médiation du détective donne la possibilité de délimiter ses ancrages pour mieux développer une conscience anticipante, transformante, migrante.

\section{Des perspectives cliniques}

Même si nous devons poursuivre la recherche en expérimentant d'autres voies narratives n'offrant pas tant aux enfants des occasions de se dire et de s'écrire mais des ressources langagières afin de jouer avec des éléments épars de leur vie, de "créer un personnage qui n'est pas exactement eux $x^{23}$, les résultats déjà obtenus ouvrent des voies pour une meilleure insertion scolaire des enfants issus des milieux migrants, dans une perspective clinique d'éducation et de formation. La force de la narration, c'est d'abord de susciter une écoute, une prise en compte par les pairs, par l'institution scolaire, de la personne et pas seulement de l'élève, ce qui n'est pas si courant aujourd'hui. Non seulement, les enfants mettent à jour des fragments de leur vécu qu'ils ont envie de partager, mais ils sont aussi confrontés à d'autres récits d'enfants susceptibles de résonner en eux. Le récit peut permettre cette appropriation de l'expérience des autres car il laisse celui qui le reçoit établir ses propres liens avec sa situation et ses réflexions, il n'impose pas une lecture mais des possibles, dont chacun s'empare ou non ${ }^{24}$. La force de la narration, c'est ensuite d'ouvrir des espaces d'entre-deux : entre l'enfant et l'enfant, entre l'enfant et l'adulte, entre la réalité et la fiction, mais aussi entre l'histoire familiale et l'histoire scolaire : "Madina habite au Mans. Elle a six sœurs, un frère. Ils sont tous nés à Djibouti. Tous les matins la famille se lève entre 6 et 7 heures. Personne ne les emmène à l'école, à part la plus petite." Le détective de Madina jette un pont entre la famille et l'école ; à travers cette remarque "personne ne les emmène à l'école”, Madina appelle peutêtre de ses vœux une implication plus importante de sa famille, elle réalise peut-être aussi qu'elle est grande et qu'elle va devoir transformer elle-même son histoire. "Le récit ou l'écriture modifient en retour la vie, même quand ils tentent de l'exprimer au plus juste : ils peuvent la figer, l'endormir, l'exacerber, la trahir, parfois la réduire aux mots pour la dire... En tout cas, ils la perturbent et la (dés)orientent ${ }^{25}$."

La force de la narration, c'est enfin de faire de celui qui se risque au récit un "auteur", qui par le moyen de l'écriture va donner un mouvement à la vie. Pour les enfants d'origine étrangère, la fiction peut constituer une sphère d'invention de leur inscription personnelle dans la société, et notamment une voie de conciliation de leur histoire familiale et de leur histoire scolaire. Plutôt que d'enfouir la migration, elle lui confère une forme qu'elle "refigure". 